\title{
Démantèlement de l'installation nucléaire de base 106 (LURE)
}

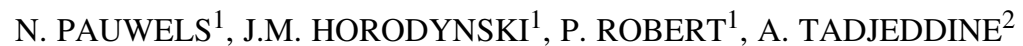

(Manuscrit reçu le 27 février 2013, accepté le 15 mai 2013)

RÉSUMÉ Dans le but d'obtenir le déclassement de l'installation nucléaire de base 106 (INB 106), trois de ses accélérateurs ont été arrêtés puis démantelés et un autre modifié pour passer sous le seuil de classement INB. Le démantèlement a été mené sur la base d'une stratégie de démontage mécanique sans découpe. Les éléments de génie civil peu activés ont pour la très grande majorité été laissés en place sans traitement avec des mesures compensatoires de protection du public et de l'environnement. Le résultat global de ces opérations est un gain en termes de coût et de délais et une importante diminution des risques notamment radiologiques, l'impact du site à l'état final restant acceptable.

ABSTRACT Decommissioning of the LURE Nuclear Facility.

With the goal of obtaining the decommissioning of the LURE nuclear facility, three of its accelerators were dismantled and another was modified to be below the threshold of "Installation Nucléaire de Base" status. Operations were carried out with the strategy of mechanical dismantling with no cutting process. As the civil engineering radioactivity level was low, a great majority of it has been left in place with no processing, but compensatory measures have been taken for public and environmental protection. The overall result of these operations is a gain in both cost and operating time. They also contribute to a significant decrease in the risks, including radiological ones. The radiological impact after decommissioning remains acceptable.

Keywords: radiation protection / accelerator / decommissioning / dismantling / waste management

\section{Introduction}

L'histoire des accélérateurs et des anneaux de collision et de rayonnement synchrotron d'Orsay se confond avec celle de la physique des particules et des hautes énergies d'une part, celle de l'utilisation du rayonnement synchrotron produit par ces particules pour la caractérisation de la matière d'autre part.

Commencée en 1955 à l'initiative des physiciens de l'École Normale Supérieure, la construction du grand accélérateur linéaire (LINAC) fut réalisée par tranches successives de $250 \mathrm{MeV}$, visant à accélérer les électrons jusqu'à 1,3 GeV

\footnotetext{
CNRS/Unité de Démantèlement de l'INB 106 (LURE), Centre Universitaire Paris Sud, Bâtiment 201 porte 1, 91898 Orsay Cedex, France.
}

2 Laboratoire de Chimie Physique, Centre Universitaire Paris Sud, Bâtiment 349, 91898 Orsay Cedex, France. 
(2,3 GeV en 1968) sur une longueur de 300 mètres donnant sur l'Igloo. Il était un des accélérateurs le plus puissant du monde, dédié d'abord à l'étude de la structure de la matière par diffusion des électrons puis, à partir de 1961, utilisé comme injecteur d'électrons ( $\left.\mathrm{e}^{-}\right)$et de positons $\left(\mathrm{e}^{+}\right)$dans des anneaux de collision (Marin 1960, 2009). Après la première mondiale qu'avait été l'observation des collisions $\mathrm{e}^{-} / \mathrm{e}^{+}$ (décembre 1963) sur le petit anneau Ada prêté par les laboratoires de Frascatti (Italie) (Bernardini, 2004), deux anneaux de collision furent construits : ACO (Anneau de Collision d'Orsay) achevé en 1965, exploité de 1966 à 1988 (Dagneaux et al., 1975) et reconverti en musée, inscrit à l'inventaire supplémentaire des monuments historiques par arrêté du 7 mars 2002, et DCI (Dispositif de Collision dans l'Igloo), composé de deux anneaux superposés, se croisant en deux points, pour réaliser des collisions $\mathrm{e}^{-} / \mathrm{e}^{+}$à $1,83 \mathrm{GeV}$, construit en 1977 (Lemonnier et al., 1978). Ces deux anneaux - conçus à l'origine pour la physique des hautes énergies - ont été ouverts aux utilisateurs du rayonnement synchrotron, avant d'être exploités uniquement comme sources synchrotron, les expériences de physique des hautes énergies se faisant désormais dans les accélérateurs du CERN, de Hambourg ou de Los Alamos. Les installations d'Orsay et leur personnel d'exploitation sont alors transférés en 1985 du Laboratoire de l'Accélérateur Linéaire (LAL) au Laboratoire pour l'Utilisation du Rayonnement Electromagnétique (LURE), exploité conjointement par le CNRS, le CEA, le Ministère de l'éducation nationale, de l'enseignement supérieur et de la recherche et l'Université Paris-Sud.

Un troisième anneau, Super-ACO, conçu et optimisé pour la production du rayonnement synchrotron dans la gamme spectrale allant de l'infrarouge aux $\mathrm{X}$ tendres est en exploitation dès 1987 (Sommer et al., 1988). Enfin un laser à électrons libres (CLIO : Centre Laser Infrarouge d'Orsay), équipé de son propre accélérateur a été mis en exploitation en 1992 (Glotin et al., 1992 ; Peremans et al., 1994). La construction de CLIO, classé INB du fait des caractéristiques de son accélérateur, constitue la principale modification de l'INB 106 et son exploitation a été autorisée par décret (JORF, 1992). On notera également la présence, dans le périmètre de l'INB 106, de l'accélérateur d'électrons PHIL (9 MeV et $300 \mathrm{~mW}$ ) qui ne relève pas du régime des INB.

LURE a été à l'origine de beaucoup de découvertes et inscrivait son activité dans une dynamique nationale, européenne et internationale. Le laboratoire a beaucoup œuvré pour la création du synchrotron européen ESRF localisé à Grenoble et pour le remplacement de ses machines devenues obsolètes par la nouvelle installation SOLEIL (Source Optimisée de Lumière d'Energie Intermédiaire du LURE).

L'installation a été exploitée sans incident classé à un niveau supérieur à 1 dans le cadre de la réglementation des INB. Le LINAC, DCI et Super-ACO ont été 
définitivement arrêtés fin 2003 et leur démantèlement immédiatement entrepris par le CNRS, exploitant nucléaire de l'INB 106. Le démantèlement a été confié à une Unité Propre de Service, qui a commencé sa mission dès l'arrêt des accélérateurs. Seule l'exploitation du laser à électrons libres CLIO et de l'accélérateur PHIL se poursuit, CLIO ayant été modifié dans le cadre du démantèlement pour passer sous le seuil INB.

\section{Présentation générale du démantèlement de l'INB 106}

Dans cet article, nous présenterons plus particulièrement les opérations réalisées dans le cadre du décret «démantèlement» (JORF, 2009). Les activités concernées par ce décret étaient de deux types :

- le démantèlement et l'assainissement des installations du LINAC, de l'Igloo DCI et du tunnel Super-ACO ;

- la modification des caractéristiques de l'accélérateur CLIO pour l'empêcher de délivrer un faisceau d'électrons d'énergie supérieure à $50 \mathrm{MeV}$ et de puissance supérieure à $1 \mathrm{~kW}$ (seuil de classement INB (JORF, 2007)).

\subsection{Le contexte réglementaire}

Le démantèlement de l'INB 106 s'est déroulé durant une période qui a vu une évolution importante de la réglementation relative aux INB avec notamment la loi TSN de juin 2006 (JORF, 2006) et ses décrets d'application. La préparation du démantèlement débutée en 2002, soit 2 ans avant l'arrêt des accélérateurs, a été principalement menée sur la base de la note SD3-DEM-01 de l'ASN. Dans ce cadre, il était prévu une phase de cessation définitive d'exploitation (CDE) couverte par le décret d'exploitation suivie de la mise à l'arrêt définitif et du démantèlement (MAD-DEM) autorisés par un décret unique. Notre dossier de CDE a été instruit conformément à la note SD3-DEM-01 et le dossier de demande de MAD-DEM, conçu dans l'esprit de cette note et transmis en janvier 2006, a été instruit durant le changement de réglementation et sa mise en application. Cette situation, sans avoir nécessité la refonte de notre dossier, a eu de fait une influence sur son instruction et a engendré des contraintes sur la gestion de l'état final pour lequel il était prévu de ne pas procéder à un assainissement total. Malgré tout, la décision du CNRS de procéder au démantèlement dès l'arrêt des installations en décembre 2003 et son programme de réalisation étaient en bon accord avec la politique de l'ASN $(2009,2010)$.

\subsection{Retour d'expérience d'installations similaires}

Lors de la préparation du démantèlement, la prise en compte du retour d'expérience d'autres installations (Report EUR, 1999) a apporté de nombreuses 
informations qui ont alimenté la réflexion sur la stratégie à mettre en place. Le champ d'exploration ne s'est pas limité aux seuls accélérateurs ; des données plus généralistes (OCDE, 2005) ont également contribué à l'élaboration du projet de démantèlement.

\subsection{Description des installations concernées par le démantèlement}

\subsubsection{Super-ACO}

L'accélérateur Super-ACO était situé au centre d'un hall d'expérience de $2000 \mathrm{~m}^{2}$ dans un tunnel octogonal délimité par des murs et des dalles de toit en béton. Le sol de ce hall est constitué d'une dalle en béton et le plafond d'une couverture métallique.

\subsection{2. $D C I$}

DCI était situé dans l'Igloo, local cylindrique de $1300 \mathrm{~m}^{2}$ de surface reposant sur une dalle de béton et recouvert d'un dôme. La hauteur maximale de plafond (dôme formé d'une dalle de béton) est de $22 \mathrm{~m}$. La salle de l'Igloo abritait également les départs des lignes de rayonnement synchrotron qui aboutissaient dans les bâtiments connexes abritant les halls d'expériences.

\subsubsection{LINAC}

Le LINAC s'étend sur une longueur de $300 \mathrm{~m}$ dans une tranchée divisée en deux parties : la tranche électrons et la tranche positons. Ces deux sections sont séparées par la zone du convertisseur R5 où étaient produits les $\mathrm{e}^{+}$. La tranchée du LINAC est délimitée latéralement par des parois en béton d'une épaisseur de $2 \mathrm{~m}$; des dalles de béton, réparties sur trois couches, forment le plafond. La tranchée positons est longée parallèlement par une galerie technique d'une longueur approximative de $110 \mathrm{~m}$.

\subsubsection{L'accélérateur CLIO}

L'installation CLIO comprend un accélérateur d'électrons qui peut délivrer un faisceau de $60 \mathrm{MeV}$ et de $6 \mathrm{~kW}$, ce qui le classe dans la catégorie INB. Sa modification pour limiter son domaine de fonctionnement et l'empêcher d'aller au-delà de $50 \mathrm{MeV}$ et $1 \mathrm{~kW}$ est une des opérations de la MAD-DEM.

\section{4. État initial}

L'état initial est celui qui a précédé immédiatement le début des opérations de MAD-DEM, il a été atteint en décembre 2008 après les opérations conduites au cours de la phase de CDE. Ces travaux ont été effectués dans le cadre des 
opérations autorisées par le référentiel de sûreté d'exploitation de l'installation en utilisant les méthodes, les techniques, les procédés et les matériels couramment employés pendant la période d'exploitation normale.

Les opérations réalisées durant la $\mathrm{CDE}$ ont permis de démonter l'ensemble des matériels et matériaux liés aux expériences scientifiques :

- le hall d'expériences de Super-ACO (20 lignes de lumière et 20 postes d'expérience) ;

- les halls d'expériences de DCI (6 lignes de lumière et 20 postes d'expérience);

- le hall des modulateurs.

Au début des travaux de MAD-DEM, la situation était la suivante :

- toutes les opérations de CDE étaient achevées (le LINAC, DCI et Super-ACO étaient définitivement arrêtés, les halls d'expériences de DCI et du hall SuperACO étaient vidés de tout matériel et le hall des modulateurs et la galerie technique de l'accélérateur LINAC étaient démantelés);

- le zonage radiologique et le zonage déchets de l'installation étaient mis à jour ;

- les opérations de désamiantage, la rénovation du réseau électrique de l'installation étaient achevées et la quasi-totalité des PCB évacués ;

- le périmètre de l'INB était clôturé et sécurisé ;

- des cartographies radiologiques avaient été réalisées sur l'ensemble des installations.

L'état radiologique initial de l'installation, à l'issue des opérations de CDE était le suivant :

- les trois accélérateurs (LINAC, DCI et Super-ACO) avaient fait l'objet d'une cartographie radiologique. Le niveau maximal du débit de dose $(130 \mu \mathrm{Sv} / \mathrm{h})$ était localisé exclusivement au niveau de la lentille adiabatique (zone convertisseur R5) ;

- seuls les murs, sols et dalles de couverture de la tranchée du LINAC au niveau des convertisseurs étaient activés. Ces zones avaient fait l'objet de calculs d'activation, de mesures du débit de dose et de caractérisation radiologique de carottes de béton. Les principaux radionucléides mis en évidence sont ${ }^{152} \mathrm{Eu}$, ${ }^{154} \mathrm{Eu},{ }^{137} \mathrm{Cs},{ }^{60} \mathrm{Co}$ et ${ }^{55} \mathrm{Fe}$ avec des activités inférieures à $300 \mathrm{~Bq} / \mathrm{g}$ en 2007 ;

- l'état radiologique de l'installation CLIO n'a pas été pris en compte dans ce cadre puisqu'elle reste en exploitation ; seule la modification de ses caractéristiques a été incluse dans le programme de MAD-DEM.

\section{5. État final}

L'objectif final de la MAD-DEM est d'obtenir le déclassement de l'INB 106 et de restituer les locaux libérés au propriétaire (l'Université Paris-Sud). À l'issue de ces 
opérations de MAD-DEM, les locaux des accélérateurs LINAC, DCI et Super-ACO ont été vidés, l'accélérateur CLIO ne peut plus produire de faisceau d'électrons d'énergie supérieure à $50 \mathrm{MeV}$ et d'une puissance supérieure à $1 \mathrm{~kW}$. Les dalles activées qui composaient le plafond des zones des convertisseurs ont été évacuées durant les opérations de démantèlement et remplacées par des dalles neuves.

Dans la configuration finale de l'INB 106, il reste dans l'installation une faible radioactivité résiduelle au niveau des murs de la tranchée du LINAC essentiellement localisée au niveau du convertisseur R5.

\section{Déroulement du démantèlement}

\subsection{Préambule}

\subsubsection{Démontage partiel de Super-ACO hors MAD-DEM}

Plusieurs éléments constituant l'anneau Super-ACO, dont le démontage était programmé pour la phase de MAD-DEM, ont fait l'objet de demandes de cession avant la parution du décret MAD-DEM en 2009 (JORF, 2009). La plus conséquente par la quantité de matériel et l'importance du projet était celle du CERN qui souhaitait récupérer les 32 quadrupôles de l'anneau et des éléments du transport de faisceau pour les réutiliser dans le cadre du projet CLIC. Ces demandes ont été acceptées par le Conseil d'administration du LURE (la cession au CERN constitue une partie de la contribution de la France à ce projet international). Ces cessions de matériel ont été réalisées après l'obtention d'une autorisation de l'ASN obtenue sur la base d'un dossier spécifique. Ce dossier a été élaboré avec deux objectifs principaux qui sont d'une part, mettre en évidence les bénéfices en terme de valorisation des déchets et de réduction des coûts et d'autre part, tester la méthodologie choisie pour le démantèlement des accélérateurs et dégager un retour d'expérience pour le futur chantier.

Le démontage des éléments listés ci-dessus a représenté une opération importante en raison de la quantité de matériel cédée et de l'ampleur des travaux nécessaires à leur démontage. Cette opération a permis de mettre en place une méthodologie de travail pour le futur démantèlement des accélérateurs et de tester cette méthodologie sur le «terrain».

Les enseignements que nous avons tirés de cette opération effectuée en 2006 ont montré que l'organisation du chantier constitue une des principales clefs de la réussite. Il faut notamment :

- rendre l'accès aux éléments à démonter le plus aisé possible pour diminuer les risques d'accidents, le temps de travail et par conséquent le temps d'exposition aux rayonnements ionisants ; 
- adapter les accès au chantier pour permettre l'évacuation des pièces volumineuses ;

- concevoir et fabriquer des outillages complémentaires aux moyens de levage et apparaux disponibles dans l'installation;

- accorder une attention particulière à tous les travaux de déconnexion de l'alimentation électrique, de déconnexion des fluides et de préparation du chantier ;

- repérer et identifier les éléments avant le démontage ;

- tracer et gérer les éléments et les déchets démontés dès leur production ;

- évacuer le plus rapidement possible les éléments vers une zone de transit pour ne pas encombrer la zone de travail ;

- dégager des zones tampons pour les manœuvres d'éléments lourds et encombrants, l'entreposage des éléments démontés, la caractérisation radiologique des éléments démontés et leur conditionnement pour le transport ;

- respecter la non interférence entre les activités situées en zone à déchets conventionnels et celles situées en zone à déchets nucléaires ;

- maîtriser et contrôler les entrées et sorties des personnels et des matériels ;

- veiller à maintenir l'exposition des intervenants aux rayonnements ionisants au niveau le plus faible possible.

L'expérience du démontage partiel de Super-ACO a permis également de montrer que les accélérateurs peuvent être démontés mécaniquement sans avoir à réaliser de découpe par point chaud. Par exemple les aimants les plus gros de Super-ACO (10 tonnes) ont pu être déculassés et séparés en deux éléments plus facilement manipulables. Les seules découpes mises en œuvre ont été réalisées avec des coupe-boulons et un coupe-tubes sans produire de contamination.

\subsubsection{Stratégie d'assainissement des murs}

L'état du génie civil et le niveau de l'activité massique des parois activées et son évolution au cours du temps ont fait l'objet de plusieurs études qui ont démontré l'intérêt de ne pas réaliser un assainissement complet des murs et des sols activés. La note SD3-DEM-02 n'a donc pas été appliquée ; les deux principaux arguments étaient d'une part, le risque important de fragilisation du bâtiment en cas d'assainissement, d'autre part le faible niveau d'activité résiduelle et sa décroissance relativement rapide dans le temps, comparé au risque radiologique généré par les travaux. On notera que les murs concernés sont constitués de 2 parois bétons avec au milieu du sable très fin.

Par conséquent, il a été décidé de ne procéder qu'à l'évacuation des dalles de couverture activées et de les remplacer par des dalles neuves non radioactives. En contrepartie, les zones non assainies seront grevées de servitudes d'utilité publique 
dès le déclassement de l'INB garantissant la surveillance, la traçabilité et la prise en compte du risque radiologique en cas d'intervention ou de travaux dans la zone.

\subsection{Méthodologie retenue pour le démantèlement}

À l'exception des tuyaux de refroidissement et des câbles électriques, nous avons vu précédemment que tous les éléments composant les accélérateurs à démanteler pouvaient être démontés mécaniquement. La méthodologie retenue pour les opérations de MAD-DEM a donc été de procéder au démontage mécanique jusqu'à l'obtention d'éléments conditionnables conformément aux spécifications de l'ANDRA. Les pièces élémentaires les plus importantes en volume et en poids qui entraient dans la constitution des accélérateurs étaient des poutres de support en aluminium de 6 mètres de longueur (sections accélératrices du LINAC) et des culasses d'environ 25 tonnes (parties d'aimants de déviation de 30 tonnes de l'anneau DCI). Suite à des réunions avec notre chargé d'affaire à l'ANDRA, nous avons défini des solutions pour une prise en charge par l'ANDRA sans avoir à les découper. Le démantèlement a ainsi pu être mené sans avoir de découpe par point chaud. La découpe des câbles électriques et des tuyaux a été faite avec des coupeboulons et des coupe-tubes qui ne produisent pas de déchet contaminant. Dans le cas où l'utilisation de coupe-tubes n'était pas possible, les découpes ont été réalisées avec une scie de type sabre ou crocodile. Ces scies ne produisent que très peu de chaleur et pas d'étincelles et les résidus issus de la découpe de métaux avec ces outils se présentent sous forme de limaille ; de plus, les projections de matière sont très localisées et aucune contamination atmosphérique n'est produite. Chaque découpe devait être autorisée par le chef d'INB et effectuée sous le contrôle du $\mathrm{SCR}$, conformément à une procédure spécifique. Le risque de contamination ainsi écarté, la gestion du risque radiologique et des déchets a été considérablement simplifiée avec pour effet une diminution du coût et de la durée du chantier.

\subsection{Description des travaux}

\subsubsection{Contraintes}

Pour mener à bien les opérations de démontage et d'évacuation, notre démarche a pris en compte le retour d'expérience du démontage partiel de Super-ACO (voir paragraphe 3.1.1).

\subsubsection{Pièces à manipuler}

Les pièces manipulées lors des opérations de démontage étaient de plusieurs types :

- des protections biologiques en béton (blocs et dalles de toit); 
TABLEAU I

Les tâches élémentaires et leur programmation.

Elementary task order.

\begin{tabular}{|ccccc}
\hline Tâches élémentaires & $\begin{array}{c}\text { Phase 1 } \\
\text { Super-ACO }\end{array}$ & $\begin{array}{c}\text { Phase 2 } \\
\text { DCI }\end{array}$ & $\begin{array}{c}\text { Phase 3 } \\
\text { LINAC }\end{array}$ & $\begin{array}{c}\text { Phase 4 } \\
\text { convertisseur }\end{array}$ \\
\hline Dépose des dalles de toit en béton & 1 & $\mathrm{X}$ & 1 & 1 \\
\hline Dépose des protections biologiques (parois amovibles en béton) & 5 & $\mathrm{X}$ & $\mathrm{X}$ & $\mathrm{X}$ \\
\hline Déconnexion des réseaux de fluides & 3 & 2 & 3 & 3 \\
Dépose des éléments périphériques & 2 & 1 & 2 & 2 \\
\hline Démontage des éléments constituant les accélérateurs & 4 & 3 & 4 & 4 \\
\hline Mise en place de protections biologiques & $\mathrm{X}$ & $\mathrm{X}$ & $\mathrm{X}$ & 5 \\
\hline
\end{tabular}

- des protections biologiques en plomb (écrans constitués de briques de plomb) ;

- les éléments des accélérateurs LINAC, Super-ACO et DCI ;

- des réseaux de fluides divers (circuits électriques, circuits d'eau de refroidissement, air comprimé...) ;

- des éléments périphériques (baies électroniques, boîtiers d'alimentation, pompe à vide...).

Les pièces lourdes et volumineuses ont été manipulées avec les ponts roulants disponibles et dans certains cas avec des chariots élévateurs.

\subsubsection{Tâches élémentaires}

Les opérations de démontage étaient basées sur des tâches élémentaires souvent répétitives décrites dans le tableau I.

Les activités mises en œuvre pour les tâches élémentaires présentées dans le tableau I sont :

- des démontages et des travaux de manutention ;

- des découpes ;

- des contrôles radiologiques de premier niveau et des marquages ;

- l'élimination (si origine ZDC) ;

- des transferts vers les aires de transit (si origine ZDN) ;

- des conditionnements pour l'expédition à l'ANDRA (si origine ZDN).

\subsection{Phasage du démantèlement}

Le démantèlement et l'assainissement ont été divisés en 4 phases correspondant à des zones délimitées par des barrières physiques renfermant les accélérateurs et les équipements à démanteler. Ces zones, identifiées sur le schéma 1, sont le tunnel Super-ACO, DCI, le LINAC (section située entre les canons à électrons et le 


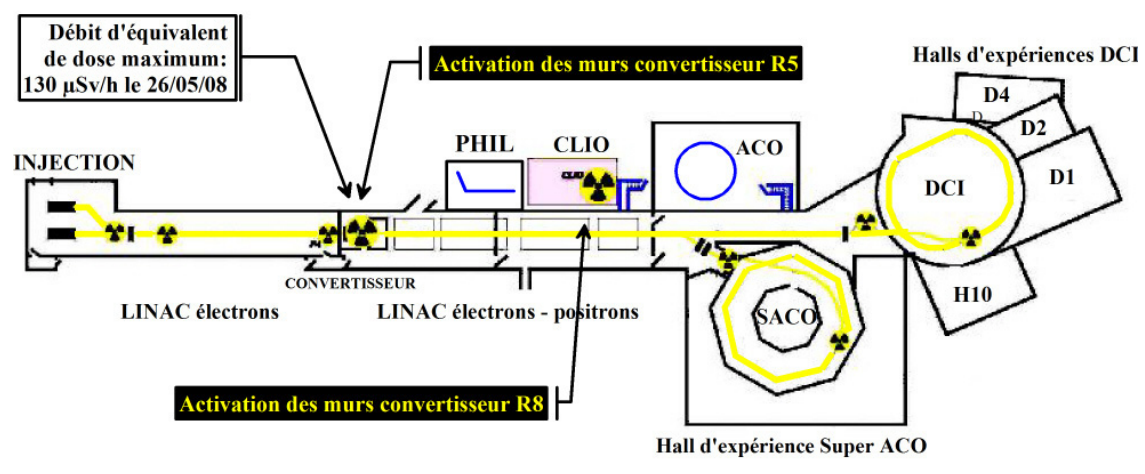

Schéma 1 - Bilan de l'activation du site.

Area residual radioactivity.

convertisseur et section entre le convertisseur et les anneaux synchrotrons) et le convertisseur R5. L'ordre de démontage était le suivant : 1-Super-ACO, 2-DCI, 3-LINAC et 4-convertisseur R5. Seules quelques opérations de la phase 3 et la totalité de la phase 4 présentaient un risque significatif d'exposition externe. Dans le but de minimiser l'exposition des travailleurs, ces phases ont été programmées en dernier pour bénéficier de la décroissance des radioéléments, ces phases étant par ailleurs sans impact radiologique sur les autres. Dans le cas du convertisseur, les travaux n'ont été autorisés qu'après la validation par le SCR et le chef d'INB d'une étude de radioprotection et la rédaction d'un protocole pour minimiser l'exposition des intervenants. Pour chacune des phases, nous avons déterminé un ordre de réalisation des tâches élémentaires adapté à la configuration du chantier. Afin d'éviter toute interaction entre les différentes phases et dans le but d'optimiser la traçabilité des déchets produits, nous avons défini les contraintes suivantes :

- les secteurs géographiques de chaque phase étaient distincts et délimités par des barrières physiques ;

- les zones de transit pour l'entreposage étaient différentes pour chaque phase ou devaient être vidées avant de commencer la phase suivante ;

- les phases n'ont pas été menées en parallèle.

\subsection{Objectif sécurité, sûreté et radioprotection}

Les objectifs en matière de sécurité classique, de sûreté et de radioprotection étaient les suivants :

- prévention de tout accident corporel sur les personnes intervenant dans l'installation ; 
- rejets liquides et gazeux dans l'environnement : nul (site et extérieur du site). L'INB 106 n'a pas d'autorisation spécifique de rejet. Tout est mis en œuvre pour maintenir le « rejet zéro »;

- contamination des locaux : nulle ;

- contamination interne : nulle ;

- contamination lors de découpe : nulle ;

- irradiation externe : inférieure à la limite publique de $1 \mathrm{mSv}^{-\mathrm{an}^{-1}}$;

- recherche des filières de valorisation ou d'élimination des déchets.

L'étude d'impact radiologique des opérations de MAD-DEM de l'INB 106 (LURE) a permis de faire une première évaluation des doses. Celle-ci prévoyait que l'équivalent de dose absorbé pour l'ensemble des opérations serait de $10,3 \mathrm{mSv}$ ce qui reste très raisonnable en regard de la réglementation en vigueur.

\subsection{Gestion des déchets}

\subsubsection{Identification des déchets}

Le risque de voir un déchet, radioactif ou potentiellement activé, évacué dans une filière non appropriée a été minimisé par la mise en œuvre d'un double marquage. Le premier avant les opérations de démontage sur tous les éléments destinés aux déchets et le second sur tous les déchets dès leur production. L'origine des déchets était identifiée par un sigle de couleur apposé soit directement sur le déchet, soit sur le conteneur, le code couleur reprenant les couleurs des panneaux du zonage déchets de l'INB 106. De plus, tous les déchets (ou colis de déchets) sortant de zone à déchet nucléaire $(\mathrm{ZDN})$ ont été considérés comme radioactifs. Par conséquent, préalablement à leur sortie de ZDN, ils ont été impérativement identifiés et ont fait l'objet d'une mesure du débit d'équivalent de dose au contact (contrôle de niveau 1 - CN1). Le résultat et la date de la mesure étaient inscrits de façon lisible sur une étiquette autocollante de couleur jaune comportant un trèfle (idiogramme de la radioactivité) collée sur le déchet dès sa production.

En complément, l'identification et la traçabilité des colis de déchets, y compris les pièces unitaires, ont été mises en œuvre sous la forme d'un système informatique basé sur une identification par code barre.

\subsubsection{Déchets issus de ZDC}

Tous les déchets issus de zones à déchets conventionnels (ZDC) produits dans l'INB ont été évacués vers les filières adaptées au type de déchets et autorisées sous la rubrique ICPE 2799. 
Avant leur évacuation du site, tous ces déchets ont subi un contrôle radiologique de troisième niveau $(\mathrm{CN} 3)$, réalisé par un agent du $\mathrm{SCR}$ au portique de contrôle de détection de l'installation.

\subsubsection{Déchets issus de ZDN}

Les déchets issus de ZDN ont été gérés par le SCR qui a organisé le conditionnement des déchets radioactifs en fonction des critères requis par l'exutoire autorisé (ANDRA). Il a calculé les indices IRAS (Indices Radiologiques d'Acceptation en Stockage) pour les colis et a effectué les contrôles radiologiques de premier et deuxième niveaux (CN2 caractérisation radiologique) sur les colis. Le SCR supervisait les opérations d'expédition vers la filière autorisée, renseignait les BSDR et effectuait les contrôles radiologiques relatifs aux opérations de transports de matières radioactives (classe 7) conformément à la réglementation.

\subsection{Choix du prestataire}

Les activités de démantèlement étant très différentes de celles de l'exploitation, la nature des risques a également fortement évolué. Le risque d'exposition externe, très important en fonctionnement, diminue fortement à l'arrêt. Par exemple au niveau du convertisseur R5, accélérateur en fonctionnement la dose létale était atteinte en quelques secondes alors qu'au même endroit en phase démantèlement, le débit de dose maximal était de $130 \mu \mathrm{Sv} / \mathrm{h}$. L'étude des risques du démantèlement a finalement montré que le risque majeur était l'accident de manutention. Cette conclusion nous a amenés à faire le choix stratégique de privilégier comme prestataire pour le démantèlement, une société spécialisée en mécanique et manutention industrielle sans expérience avérée en radioprotection. Ce choix a eu deux conséquences importantes sur le déroulement du chantier. La première est qu'il a fallu former le personnel intervenant à la radioprotection et que le SCR de l'INB s'implique dans un suivi systématique et en temps réel de toutes les activités présentant un risque radiologique. La deuxième est que la technicité de la société a amené des solutions judicieuses pour toutes les opérations de démontage et un gain de productivité qui a également engendré une diminution du temps d'intervention et donc de l'exposition aux rayonnements ionisants.

\section{Bilan}

\subsection{Radioprotection}

Lors des phases 1 à 3, les interventions des opérations ont toutes été réalisées dans des zones surveillées ; par conséquent la surveillance radiologique individuelle a 
TABLEAU II

Bilan dosimétrique du démantèlement.

Personnel monitoring assessment.

\begin{tabular}{cccc}
\hline Intervenant & $\begin{array}{c}\text { Dose efficace }(\mathbf{H p}(\mathbf{1 0})) \\
(\mathbf{e n} \boldsymbol{\mu S v})\end{array}$ & $\begin{array}{c}\text { Dose équivalente extrémités } \\
(\mathbf{H p}(\mathbf{0 , 0 7}))(\mathbf{e n} \boldsymbol{\mu S v})\end{array}$ & $\begin{array}{c}\text { Durée d'utilisation du } \\
\text { dosimètre opérationnel }\end{array}$ \\
\hline 1 & 19 & 36 & $9 \mathrm{~h} 04 \mathrm{~min}$ \\
2 & 7 & 32 & $9 \mathrm{~h} 03 \mathrm{~min}$ \\
3 & 13 & 44 & $8 \mathrm{~h} 22 \mathrm{~min}$ \\
4 & 24 & 51 & $12 \mathrm{~h} 13 \mathrm{~min}$ \\
\hline
\end{tabular}

été faite à l'aide de dosimètres passifs. Les résultats de dosimétrie et toutes les mesures sur les éléments démontés ont été conformes aux valeurs prévues lors de l'étude d'impact radiologique des opérations de démantèlement. Le bilan de la dosimétrie opérationnelle est présenté dans le tableau II. Les valeurs sont inférieures aux prévisions de l'étude d'impact des travaux de démantèlement car cette étude est un document qui présente de façon macroscopique le démantèlement et se base sur le cas le plus pénalisant. Par ailleurs, l'étude de poste spécifique réalisée en préambule à la phase 4 a permis de choisir une méthodologie plus précise, de diminuer le temps d'intervention et d'optimiser le temps d'exposition des intervenants.

Les objectifs sécurité, sûreté et radioprotection fixés ont tous été atteints à l'exception d'un accident corporel sans gravité.

\subsection{Contrôles radiologiques}

Le bilan des contrôles radiologiques présenté dans le graphique 1 inclut les données qui couvrent la période de CDE. Ces données mettent en évidence la relation qui existe entre les activités et le type de contrôle radiologique mis en œuvre. Pendant la période de CDE (2004-2008), la majorité des matériels et déchets évacués étaient issus de ZDC et ne nécessitaient qu'un contrôle en sortie de site $(\mathrm{CN} 3)$ d'où le nombre élevé de ces contrôles. En phase de MAD-DEM (2009-2010), apparait clairement une augmentation des contrôles de CN2 qui correspondent à la caractérisation radiologique des matériels et déchets évacués. Le nombre de contrôles CN2 de 2005 et 2006 n'est pas négligeable en comparaison des autres années de CDE car l'opération de démontage partiel de Super-ACO a nécessité une caractérisation radiologique de tous les matériels cédés avant leur transfert aux organismes destinataires.

\subsection{Déchets}

Comme pour les contrôles radiologiques, nous avons inclus dans la présentation des déchets conventionnels ceux de la phase de CDE (Tab. III). Les données issues 


\section{Contrôles radiologiques}

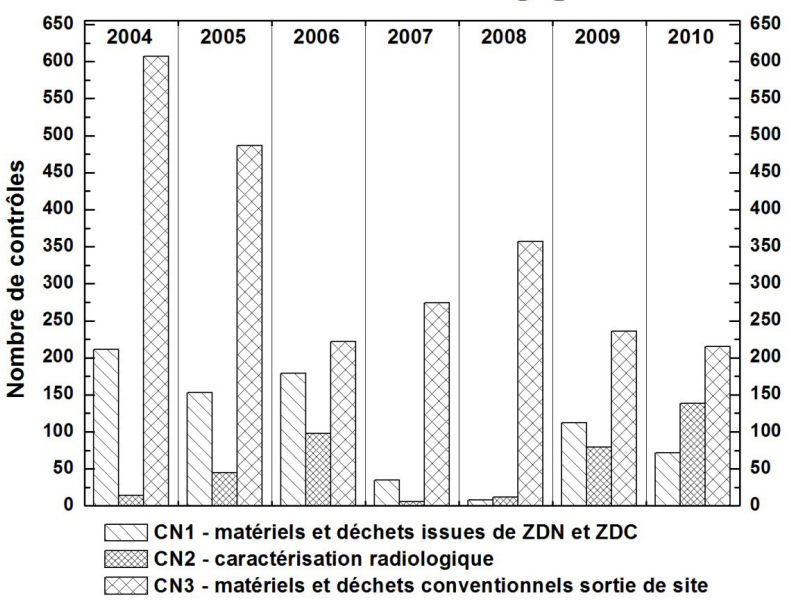

Graphique 1 - Bilan des contrôles radiologiques.

Radiological control assessment.

TABLEAU III

Bilan des déchets conventionnels.

Annual non-radioactive waste production.

\begin{tabular}{lccccccccc}
\hline & D3E & Câbles & Métaux & $\begin{array}{c}\text { Déchets } \\
\text { inertes }\end{array}$ & Bois & Plastiques & $\begin{array}{c}\text { Produits } \\
\text { Chimiques }\end{array}$ & PCB & Amiante \\
\hline $\mathbf{2 0 0 4}$ & $8 \mathrm{t}$ & $1,1 \mathrm{t}$ & $47 \mathrm{t}$ & 0 & $5 \mathrm{t}$ & $5 \mathrm{t}$ & $3,685 \mathrm{t}$ & $226 \mathrm{t}$ & $0,024 \mathrm{t}$ \\
$\mathbf{2 0 0 5}$ & 0 & $4 \mathrm{t}$ & $47 \mathrm{t}$ & 0 & $10 \mathrm{t}$ & $2 \mathrm{t}$ & $0,282 \mathrm{t}$ & 0 & 0 \\
$\mathbf{2 0 0 6}$ & 0 & $7 \mathrm{t}$ & $140 \mathrm{t}$ & 0 & $32 \mathrm{t}$ & $2 \mathrm{t}$ & $0,129 \mathrm{t}$ & $13,44 \mathrm{t}$ & $0,075 \mathrm{t}$ \\
$\mathbf{2 0 0 7}$ & $55,5 \mathrm{t}$ & $15 \mathrm{t}$ & $64 \mathrm{t}$ & 0 & $25 \mathrm{t}$ & $5 \mathrm{t}$ & $3,4 \mathrm{t}$ & $7,78 \mathrm{t}$ & $10 \mathrm{t}$ \\
\hline $\mathbf{2 0 0 8}$ & $8,5 \mathrm{t}$ & $40 \mathrm{t}$ & $125 \mathrm{t}$ & $243 \mathrm{t}$ & $10 \mathrm{t}$ & $4 \mathrm{t}$ & $0,415 \mathrm{t}$ & $25,77 \mathrm{t}$ & $38,4 \mathrm{t}$ \\
$\mathbf{2 0 0 9}$ & $17,5 \mathrm{t}$ & $10 \mathrm{t}$ & $110 \mathrm{t}$ & $215 \mathrm{t}$ & $26 \mathrm{t}$ & $5 \mathrm{t}$ & $4,218 \mathrm{t}$ & $32 \mathrm{t}$ & 0 \\
\hline $\mathbf{2 0 1 0}$ & 0 & $22 \mathrm{t}$ & $31 \mathrm{t}$ & $174 \mathrm{t}$ & $2,7 \mathrm{t}$ & $4,3 \mathrm{t}$ & 0 & $35,8 \mathrm{t}$ & $1,45 \mathrm{t}$ \\
\hline Total & $\mathbf{8 9 , 5 ~ t}$ & $\mathbf{9 9 , 1 ~ t}$ & $\mathbf{5 6 4 ~ t}$ & $\mathbf{6 3 2} \mathbf{t}$ & $\mathbf{1 1 0 , 7} \mathbf{t}$ & $\mathbf{2 7 , 3 ~ t}$ & $\mathbf{1 2 , 1 2 9} \mathbf{t}$ & $\mathbf{3 4 0 , 7 9 t}$ & $\mathbf{5 0 ~ t}$ \\
\hline
\end{tabular}

du tableau montrent également une forte production de déchets conventionnels en phase de CDE qui sont notamment dûs aux travaux d'assainissement (désamiantage, évacuation des $\mathrm{PCB}$...) qui ont été réalisés préalablement à la MAD-DEM. Ces travaux sont essentiellement du désamiantage, l'évacuation des PCB et la réhabilitation du réseau électrique. Ils sont comptabilisés dans les contrôles $\mathrm{CN} 3$ du graphique 1 . 
TABLEAU IV

Bilan des déchets nucléaires.

Nuclear waste production.

\begin{tabular}{ccccc}
\hline & Poids $(\mathbf{t})$ & Volume $\left(\mathbf{m}^{\mathbf{3}}\right)$ & $\begin{array}{c}\text { Container } \\
\text { ISO } \mathbf{1} \text { /2 hauteur }\end{array}$ & Pièces unitaires \\
\hline $\begin{array}{c}\text { Phase 1 } \\
\text { (Super-ACO) }\end{array}$ & 121 & 99 & 4 & 8 \\
Phase 2 (DCI) & 642 & 294 & 8 & 98 \\
Phase 3 (LINAC) & 261 & 430 & 21 & 8 \\
\hline Phase 4 (Convertisseur) & 35 & 28 & 4 & 0 \\
\hline TOTAL & 1059 & 851 & 37 & 114 \\
\hline
\end{tabular}

Le tableau IV détaille les masses, les volumes et les types de colis de déchets nucléaires produits pour chacune des 4 phases du démantèlement. La quantité de déchets produits lors du démontage de Super-ACO est nettement inférieure à nos prévisions initiales car plus de $60 \%$ de l'accélérateur avaient été cédés lors du démontage partiel, soit 250 tonnes de matériel valorisé.

\subsection{Incidents et non conformités}

Durant les opérations de démantèlement, 4 écarts ont été traités dans le système de management de la qualité mis en place pour le démantèlement et certifié ISO 9001. La procédure de maîtrise des non conformités prévoyait notamment l'arrêt du chantier et la mise en œuvre d'actions correctives et préventives. Ces anomalies ne rentraient pas dans les critères de déclarations des évènements significatifs auprès de l'ASN.

Deux non conformités relèvent des dysfonctionnements sur le chantier liés essentiellement au prestataire qui manquait d'expérience du travail en INB et de l'application stricte de procédure :

- non-respect de la procédure de découpe en ZDN ;

- non-respect de la procédure de gestion des déchets lors du démantèlement du LINAC.

Les deux autres non conformités, non directement liées au chantier, ont été mises en évidence à l'occasion des opérations de démantèlement :

- présence d'uranium dans un composant électronique issu de ZDC ;

- présence d'amiante dans l'Igloo.

\subsection{Impact radiologique après déclassement}

L'impact du site après déclassement de l'INB a fait l'objet d'une étude qui explore plusieurs scénarii et détermine celui qui engendre l'exposition maximale aux 
rayonnements ionisants. Les scénarii choisis sont ceux du guide IRSN (ASNIRSN, 2011) auquel nous avons ajouté la démolition du bâtiment. Les modèles de déposition, de contamination des végétaux, de l'eau et des sols sont tirés du rapport IAEA (2001). Les cas les plus pénalisants sont ceux de l'incursion sur friche et de l'occupation du bâtiment pour un usage privé qui conduisent à une exposition externe inférieure à $1 \mathrm{mSv} / \mathrm{an}$ à partir de $2020(900 \mu \mathrm{Sv} / \mathrm{an})$ et inférieure à la moitié de cette valeur dès 2030 (475 $\mu \mathrm{Sv} / \mathrm{an})$. L'exposition interne maximale $(166 \mu \mathrm{Sv} / \mathrm{an}$ en 2020 et $22 \mu \mathrm{Sv} /$ an en 2030), obtenue dans le cas du scénario de maraîchage, est 1 à 2 ordres de grandeur en-dessous de l'exposition externe.

À l'issue du déclassement, les accélérateurs PHIL et CLIO resteront en exploitation et les locaux libérés lors du démantèlement seront réaffectés à des activités de recherche expérimentale (la construction de 2 nouveaux accélérateurs est déjà programmée). Seule la tranchée du LINAC, grevée de servitudes d'utilité publique, ne pourra pas être réutilisée avant 2020. Dans ces conditions, l'impact du site sur les travailleurs et le public est quasi nul et en 2020, l'exposition externe d'un travailleur sédentaire dans la tranchée du LINAC serait au maximum de $98 \mu \mathrm{Sv} / \mathrm{an}$.

\section{Conclusion}

La préparation, l'organisation et le suivi permanent du chantier mis en place sont des éléments essentiels qui ont très largement contribué au bon déroulement des opérations dans des conditions de sécurité et de radioprotection optimales ainsi qu'au strict respect du planning et des coûts. Par exemple, la procédure de choix du prestataire pour les travaux de démantèlement a été lancée plusieurs mois avant la parution du décret, ce qui a permis de procéder à la préparation du chantier au début de l'année 2009 avant la parution du décret et d'engager le démantèlement dès sa publication au journal officiel.

De l'expérience du démantèlement de l'INB 106, on retiendra deux constats, le premier relatif à la méthodologie de démantèlement et le second sur l'état radiologique de l'état final. Nous avons montré que pour le démantèlement d'installation de type accélérateur de particules, il est possible de procéder uniquement à des démontages mécaniques sans avoir à réaliser de découpe par point chaud évitant ainsi le risque de contamination radiologique. Les éléments les plus gros peuvent être démontés et séparés en plusieurs parties facilement manipulables. Malgré la production de quelques pièces unitaires importantes en volume et en masse pour lesquelles des solutions spécifiques ont été mises en œuvre, tous les déchets ont pu être pris en charge par l'ANDRA.

La stratégie de ne pas assainir en totalité le génie civil a été choisie et validée car elle repose sur un argumentaire solidement étayé ; d'une part, nous avons 
montré que l'impact radiologique sur les travailleurs et l'environnement est faible et d'autre part, nous avons mis en œuvre des mesures permettant de s'assurer sur le long terme de la pérennité du contrôle et de la surveillance du site.

\section{RÉFÉRENCES}

ASN (2009) La politique de l'ASN en matière de démantèlement et de déclassement des installations nucléaires de base en France, Indice 0.v3 - avril 2009.

ASN Guide $n^{\circ} 6$ (2010) Mise à l'arrêt définitif, démantèlement et déclassement des installations nucléaires de base en France, version du 18/06/2010.

ASN - IRSN (2011) Guide méthodologique d'assainissement des sites potentiellement pollués par des substances radioactives, ASN - IRSN - Ministère de l'écologie, décembre 2011.

Bernardini C. (2004) Ada, the first electron-positon collider, Phys. Perspect. 6, 156-183.

Dagneaux P., Depautex C., Dhez P., Durup J., Farge Y., Fourme R., Guyon P.M., Jeagle P., Leach S., Lopez-Delgado R., Morel G., Pinchaux R., Thiry P., Vermeil C., Wuilleumier F. (1975) L'utilisation du Rayonnement en France: L'état des montages et des expériences, Les dispositifs prévus et les perspectives scientifiques, Ann. Phys., 1-65.

Glotin F., Berset J.M., Chaput R., Kergosien B., Hambert G., Jaroszynski D., Ortega J.M., Prazeres R., Velghe M., Bourdon J.C., Bernard M., Dehamme M., Garvey T., Mencik M., Mouton B., Omeich M., Rodier J., Roudier P. (1992) First lasing of the CLIO FEL. Dans : Proc. of the 3rd European Particle Accelerator Conference, 1991, Berlin, p. 620. Ed. Fontières, Gif sur Yvette.

IAEA safety report series $n^{\circ} 19$ (2001) Generic Models for use in assessing the impact of discharges of radioactive substances to the environment, Vienna.

JORF (1992) Décret no 92-622 du 2 juillet 1992 autorisant le CNRS à modifier l'installation nucléaire de base dénommée " accélérateur linéaire d'Orsay » par l'implantation d'un laser à électrons libres, Journal officiel de la République française du 8 juillet 1992.

JORF (2006) Loi 2006-686 du 13 juin 2006 relative à la transparence et à la sécurité en matière nucléaire, Journal officiel de la République française du 14 juin 2006.

JORF (2007) Décret 2007-830 du 11 mai 2007 relatif à la nomenclature des installations nucléaires de base, Journal officiel de la République française du 12 mai 2007.

JORF (2009) Décret 2009-405 du 14 avril 2009 autorisant le CNRS à achever les opérations de mise à l'arrêt définitif et à procéder aux opérations de démantèlement de 1'INB 106, Journal officiel de la République française du 16 avril 2009.

Lemonnier M., Fourme R., Rousseaux F., Kahn R. (1978) X-ray curved-crystal monochromator system at the storage ring D.C.I., Nucl. Instrum. Meth. 152, 173-177.

Marin P. (1960) Les accélérateurs explorent la matière, Vol. 4. Téléonde.

Marin P. (2009) Un demi-siècle d'accélérateurs de particules. Editions du Dauphin, Paris.

OCDE AEN 6073 (2005) Vers la réalisation d'un dossier de sûreté de démantèlement.

Peremans A., Tadjeddine A., Guyot-Sionnest P., Prazeres R., Glotin F., Jaroszynski D., Berset J.M., Ortega J.M. (1994) Absorbat vibrational spectroscopy by IR-visible sum frequency generation using CLIO-FEL: $\mathrm{CO}$ from $\mathrm{CH}_{3} \mathrm{OH}$ electrochemical decomposition of $\mathrm{Pt}$, Nucl. Instrum. Meth. A 341, 146-151.

Report EUR 19151 (1999) Evaluation of the radiological and economic consequences of decommissioning particle accelerators, march 1999.

Sommer E.M., Besson J.C., Certain P., Dael A., Damany A., Juan P., Labèque A., Level M.P., Marin P., Michaut J., Monet-Descomby C., Nghiem P., Souchet R., Zyngier H. (1988) Commissioning of Super-ACO. Dans : Proc. of the European Particle Accelerator Conference, Rome, pp. 1-46. 